\title{
The Evaluation of Eudragit Microcapsules Manufactured by Solvent Evaporation Using USP Apparatus 1
}

\author{
Sandile M. Khamanga, Natalie Parfitt, Tsitsi Nyamuzhiwa, \\ Hendrina Haidula, and Roderick B. Walker ${ }^{1}$ \\ Faculty of Pharmacy, Rhodes University, Grahamstown, South Africa 6140
}

\begin{abstract}
The objectives of this study were to prepare microcapsules containing verapamil and propranolol and to evaluate the kinetics and mechanism of drug release from the microcapsules using USP Apparatus 1.The effects of polymer concentration and polymer type on the cumulative amount of drug released were evaluated. The microcapsules were manufactured using Eudragit RS and RL polymers by solvent evaporation with the ultimate aim of prolonging drug release. Twenty-four formulations were prepared using different drug/polymer ratios. The effects of polymer type and polymer/drug ratios on the size, flow properties, surface morphology, and the release characteristics of the microcapsules were examined. The effects of drug inclusion methods on drug loading, encapsulation efficiency, and release properties of the complex microcapsules were also investigated. The formulations containing drug/polymer ratio 1:4 (w/w) were the most appropriate with respect to encapsulation efficiency (70\%), flow properties (HR = 1.2), drug loading (15-20\%), and drug release characteristics, in all cases. The release kinetics from the different formulations followed mainly a diffusion-controlled mechanism.
\end{abstract}

\section{INTRODUCTION}

$\mathrm{M}$ icroencapsulation is defined as the application of a thin coating to individual core materials that have an arbitrary particle size range between 5 and $5000 \mu \mathrm{m}(1,2)$. Microencapsulation is widely used in the pharmaceutical and other sciences to mask tastes or odors, prolong release, impart stability to drug molecules, improve bioavailability, and as multi-particulate dosage forms to produce controlled or targeted drug delivery (3-6). It is therefore a rapidly expanding technology for achieving sustained-release dosage forms.

The solvent-evaporation method of microencapsulation involves the use of emulsification of a solution containing polymer and drug with an additional medium in which the drug and polymer cannot dissolve (7). The technique is relatively simple and has been used to prepare microcapsules of a variety of compounds using several different polymeric materials $(5,8)$.

There are several formulation and process parameters that, when modified during the manufacture of microcapsules by solvent evaporation, may affect the properties of microspheres. The parameters in question include the aqueous solubility of raw material or drug to be encapsulated, the type and concentration of the dispersing agent, the polymer/drug ratio, and the stirring rate used to agitate the emulsion system formed during the manufacturing process (7). The Eudragit range of products is a series of acrylic and methacrylic acid copolymers that are

${ }^{1}$ Corresponding author. available in several different ionic forms. Eudragit RL and RS grades of polymer are insoluble in aqueous media but are permeable and have the ability to produce $\mathrm{pH}$-independent drug-release profiles. The permeability of Eudragit RS and RL polymers in aqueous media is a consequence of the presence of quaternary ammonium functional groups in their molecular structures, and the greater prevalence of these functionalities in the $\mathrm{RL}$ grade results in it being more permeable than the RS grade (9).

We focused considerable attention on the dissolution testing of the microcapsules manufactured in these studies, since in vitro dissolution testing of solid oral dosage forms is an integral part of pharmaceutical development and routine quality control monitoring of drug release characteristics (10). The USP recommends seven different types of apparatus for in vitro release testing. USP Apparatus 1 (basket) and 2 (paddle) were designed for the evaluation of immediate-release (IR) and modified-release (MR) oral formulations (11), whereas USP Apparatus 5 (paddle over disc), 6 (cylinder), and 7 (reciprocating holder) were designed for the assessment of transdermal products. USP Apparatus 3 (reciprocating cylinder) and 4 (flow-through cell) were designed for the evaluation of extended-release (ER) oral formulations (12).

In these studies, propranolol hydrochloride $(\mathrm{PHCl})$ and verapamil hydrochloride $(\mathrm{VHCl})$ were used to evaluate the potential of the solvent-evaporation microencapsulation technique to encapsulate drugs of different solubility and permeability. Although the solvent-evaporation process is a relatively simple concept, many process variables may influence the formation and properties of the microcapsules that are produced. 
The Biopharmaceutics Classification System (BCS) recommends methods for classifying oral drugs into four different categories based on their aqueous solubility and intestinal permeability (13). $\mathrm{VHCl}$, a calcium channel blocker, is widely used in the management of angina, supraventricular arrhythmias, and hypertension (14). $\mathrm{PHCl}$ is a non-selective, beta-adrenergic blocker that is used in the treatment of hypertension, angina pectoris, pheochromocytoma, and cardiac arrhythmias (15). $\mathrm{VHCl}$ and $\mathrm{PHCl}$ are $\mathrm{BCS}$ Class I drugs. Class I drugs are well absorbed and are readily soluble, and the rate-limiting step for absorption of these compounds is dissolution rate or gastric emptying rate should the dissolution rate be rapid (16). Reports of the preparation and evaluation of microcapsules manufactured using the selected drug candidates have been published (17-22); however, none have focused on a comparison of the resultant products.

The objectives of these studies were to incorporate two weakly basic, freely soluble drugs into matrix microcapsules and to investigate process parameters that affect the properties and in vitro dissolution of the drugs from microcapsules.

\section{EXPERIMENTAL}

\section{Preparation of Microcapsules}

$\mathrm{PHCl}$ and $\mathrm{VHCl}$ microspheres were prepared by a solvent-evaporation method using different quantities of Eudragit RS and Eudragit RL polymers. Approximately 0.5, $1.0,1.5,2.0,2.5$, and $3.0 \mathrm{~g}$ were accurately weighed and dissolved in $13.5 \mathrm{~mL}$ of acetone with stirring. A 500-mg quantity of the relevant drug and $50 \mathrm{mg}$ of magnesium stearate were then dispersed in the polymer solution. The resultant milky white dispersion was poured into a vessel containing a mixture of $135 \mathrm{~mL}$ of liquid paraffin and $15 \mathrm{~mL}$ of $n$-hexane and stirred for $5 \mathrm{~h}$ using a homogenizer fitted with a four-blade "butterfly" propeller with a diameter of $50 \mathrm{~mm}$ (Virtis Company, USA). Stirring was continued for $3 \mathrm{~h}$ at $500 \mathrm{rpm}$ or until the acetone was completely evaporated. The manufacturing procedure is schematically represented in Figure 1.

Following removal of the acetone, the resultant microcapsules were harvested by vacuum filtration after which they were washed four times with $25 \mathrm{~mL}$ of $n$-hexane and dried at room temperature $\left(25^{\circ} \mathrm{C}\right)$ for $24 \mathrm{~h}$.

Twenty-four batches of microcapsules with drug/ polymer ratios of $1: 1,1: 2,1: 3,1: 4,1: 5$, and $1: 6(\mathrm{w} / \mathrm{w})$ were prepared using Eudragit RS and RL as the encapsulation polymers.

\section{Size Distribution of Microcapsules}

Once dried, the microcapsules were sized by passing through a nest of standard sieves of mesh sizes \#18 $(850 \mu \mathrm{m}), 24(710 \mu \mathrm{m})$, and $80(177 \mu \mathrm{m})$. The mean particle size of each fraction was taken as the arithmetic mean size of the apertures of the preceding screen on which the particles had been retained.

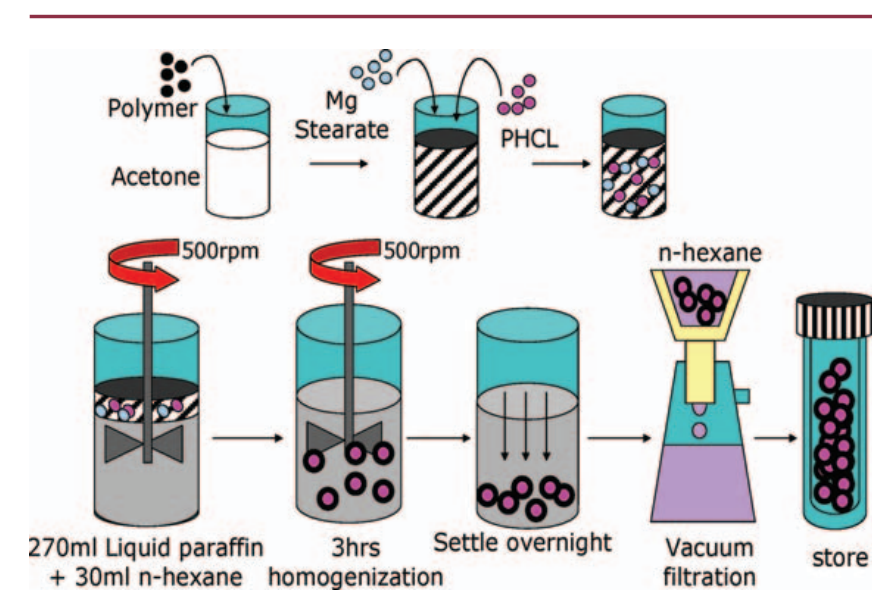

Figure 1. Manufacturing procedure used to produce microcapsules for these studies.

\section{Flow Properties of Microcapsules}

The Hausner ratio of the microcapsules manufactured using different formulations was computed according to the following relationship (23)

$$
H R=\frac{\rho_{\text {tap }}}{\rho_{\text {bulk }}}
$$

where $\rho_{\text {tap }}$ is tapped density and $\rho_{\text {bulk }}$ is bulk density.

\section{Scanning Electron Microscopy}

The shape and surface morphology of the microcapsules were investigated using scanning electron microscopy (Tescan, VEGA LMU, Czech Republic). The microcapsules were mounted onto a double-sided carbon stub that was placed on a sample disc carrier (3-mm height, 10-mm diameter) and sputter coated (Balzers Union Ltd, Balzers, Lichtenstein) with gold under vacuum (0.25 Torr). The samples were then monitored, and an image was generated using a $20 \mathrm{kV}$ electron beam.

\section{In Vitro Release Studies}

The USP basket apparatus has been used to study in vitro drug release from microspheres (24). The dissolution rate of the different drugs from the microspheres was studied in a $\mathrm{pH} 7.4$ phosphate buffer solution (100 mM). A USP Apparatus 1 (rotating basket) set to sink conditions with the basket at $100 \mathrm{rpm}$ was used to generate drug release profiles. Accurately weighed samples of the microspheres were placed in the baskets and then lowered into the dissolution medium that was maintained at $37 \pm 0.5^{\circ} \mathrm{C}$. Each formulation was tested in triplicate.

Sample aliquots were withdrawn at $0,0.5,1,2,4,8$, and $12 \mathrm{~h}$ and were filtered through a $0.45-\mu \mathrm{m}$ filter before analysis by HPLC. A volume of dissolution medium equal to what had been removed for analysis was replaced to maintain constant volume. Samples for analysis were monitored spectrophotometrically (UV-Mini 1240, UV-vis 
Spectrophotometer, Shimadzu Corporation, Japan) by recording the absorbance at $278 \mathrm{~nm}$ for $\mathrm{VHCl}$ and $280 \mathrm{~nm}$ for $\mathrm{PHCl}$. All products were observed visually to assess whether any physical changes to the particles occurred during the dissolution testing process.

Experimental results were expressed as mean \pm SD. Student's $t$-test and one-way analysis of variance (ANOVA) were applied to check significant differences in drug release from different formulations. Differences were considered to be statistically significant at $p<0.05$. The statistical software used for analysis was GraphPad Prism Software Version 4.0 (GraphPad Prism Software, San Diego, (A, USA).

\section{Drug Release Kinetics}

To investigate the mechanism of drug release from the microcapsules, the release data were analyzed using zero-order kinetic (25), Higuchi $(26,27)$, Korsmeyer-Peppas (28), Kopcha (29), and Makoid-Banakar (30) models. Modeling was performed using GraphPad Prism Software Version 4.0 (GraphPad Prism Software, San Diego, CA, USA). The software estimates the parameters of a nonlinear function that provides the closest fit between experimental observations and the non-linear function. The mathematical expressions that describe the models used to describe the dissolution curves in these studies are summarized in Table 1.The best-fit solution was identified by evaluating the coefficient of determination $\left(R^{2}\right)$, where the highest $R^{2}$ value indicates the best fit.

\section{Determination of Drug Loading, Encapsulation Efficiency, and Microcapsule Yield}

The average drug content was determined by extraction of a 20-mg sample of microcapsules with methanol. Following filtration and appropriate dilution with additional methanol, the resultant concentration was determined using UV spectrophotometry, and the percent drug loading was calculated using the following equation:

$$
\% \text { Loading }=\frac{\text { weight of drug }}{\text { weight of microparticles }} \times 100
$$

Table 1. Mathematical Representation of Models Used to Describe the Release Profiles from the Microcapsules

\begin{tabular}{ll}
\hline Model & Equation \\
\hline Zero-order & $Q_{t}=Q_{o}+K_{o} t$ \\
\hline Higuchi & $Q_{t}=Q_{o}+K_{H} t^{1 / 2}$ \\
\hline Korsmeyer-Peppas & $Q_{t}=K_{K P} t^{n}$ \\
\hline Kopcha & $Q_{t}=A t^{1 / 2}+B t$ \\
\hline Makoid-Banakar & $Q_{t}=K_{M B} t^{n} e^{(-c t)}$ \\
\hline
\end{tabular}

The encapsulation efficiency of the process was calculated using the following equation:

$$
\text { Encapsulation Efficiency }=\frac{\text { actual drug content }}{\text { theoretical drug content }} \times 100
$$

The percentage yield of the microcapsules was determined for each drug candidate and was calculated using the following equation (31):

$$
\text { Yield } \%=\frac{M}{M_{0}} \times 100
$$

where $M$ is the weight of microcapsules and $M_{0}$ is the total expected weight of drug and polymer.

\section{Assay Procedure}

The total drug content of the microcapsules was determined by dissolving accurately weighed portions of each batch of microcapsules in $100 \mathrm{~mL}$ methanol and then monitoring the UV absorbance at the relevant wavelength for each compound. At the outset, calibration curves for the two drugs were constructed, and the correlation coefficients, $r$, of the curves were established. Furthermore, the polymers did not interfere with the assay, and all samples were assayed in triplicate.

\section{RESULTS AND DISCUSSION}

During processing, it was observed that stirrer speeds of less than $500 \mathrm{rpm}$ were not sufficient to produce microcapsules, and a huge coalesced mass was obtained. This is due in part to inadequate agitation of the media to disperse the inner phase in discreet droplets within the bulk phase. At stirring speeds above $1000 \mathrm{rpm}$, the turbulence caused frothing and adhesion of the microparticles to the container walls and propeller blade surfaces, resulting in high shear and a smaller size of the dispersed droplets. Spherical microspheres were obtained at a stirring rate of $500 \mathrm{rpm}$; therefore, this speed was used during manufacture of all microcapsules.

The use of magnesium stearate as a dispersion agent decreased the interfacial tension between the lipophilic and hydrophilic phases of the emulsion and further simplified the formation of microcapsules. As the solvent evaporated, the viscosity of the individual droplets increased, and highly viscous droplets were observed to coalesce at a faster rate than they could be separated. Magnesium stearate formed a thin film around the droplets and thereby reduced the extent of coalescence, before hardening of the capsules, on collision of the droplets. The resultant microcapsules were free-flowing, and the use of magnesium stearate was deemed effective.

When 1:1 (w/w) drug/polymer concentrations were used for both the Eudragit RS and RL polymers, the quality of microcapsules formed was poor (Figures 2 and 3). These were irregularly shaped, not flowing, and presented with lots of indentation. Microcapsules were only formed when 


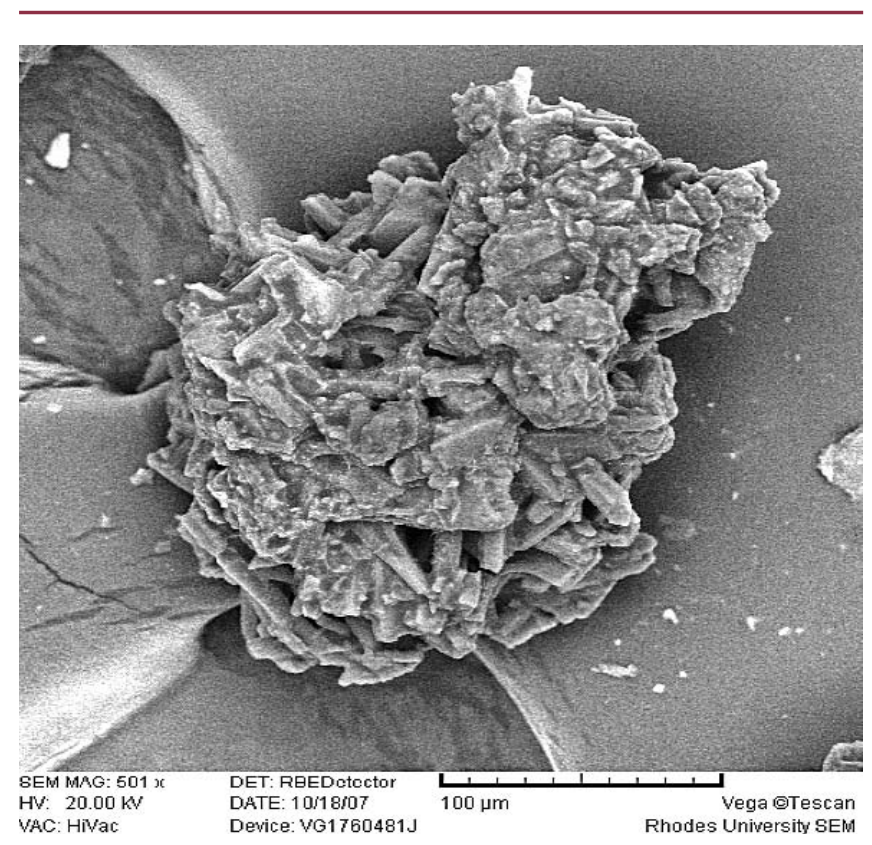

Figure 2. SEM of a PHCI/ Eudragit RS 1:1 (w/W) microcapsule.

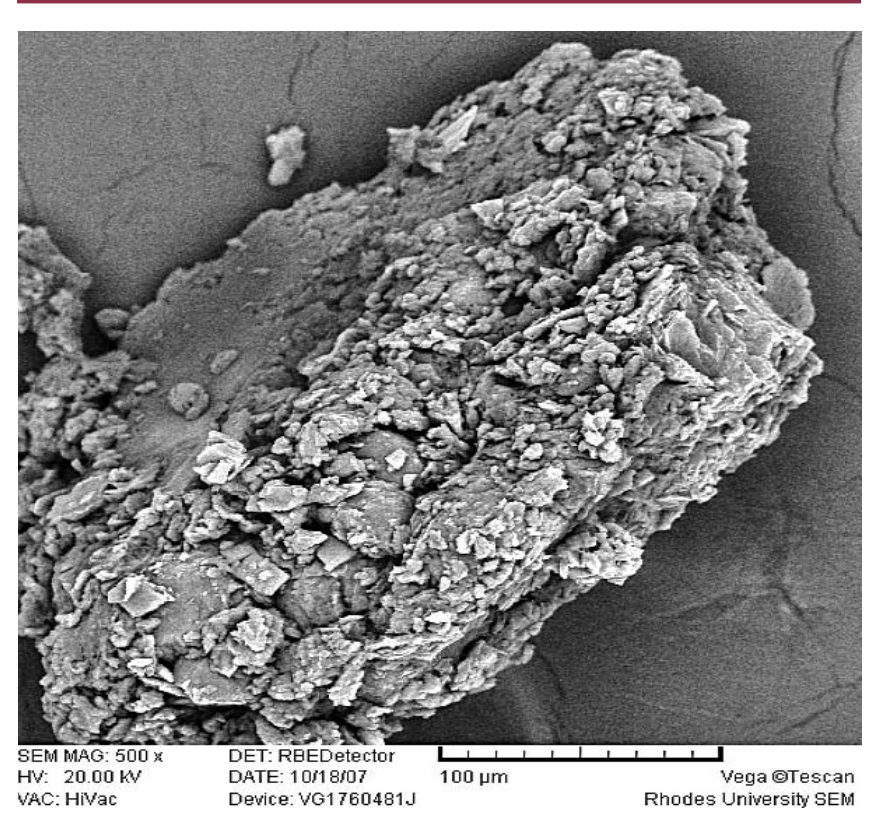

Figure 3. SEM of a VHCI/ Eudragit RS 1:1 (w/w) microcapsule.

the polymer concentration was increased to ratios of between 1:2 and 1:6 $(\mathrm{w} / \mathrm{w})$ with respect to the drug concentration.

Discrete, spherical, and uniform microcapsules were obtained with a 1:4 (w/w) drug/polymer ratio for both the $\mathrm{RS}$ and RL polymers, as can be seen in Figures $4-7$. It is also evident that the microcapsules exhibited slightly porous

Dissolution Technologies | MAY 2009

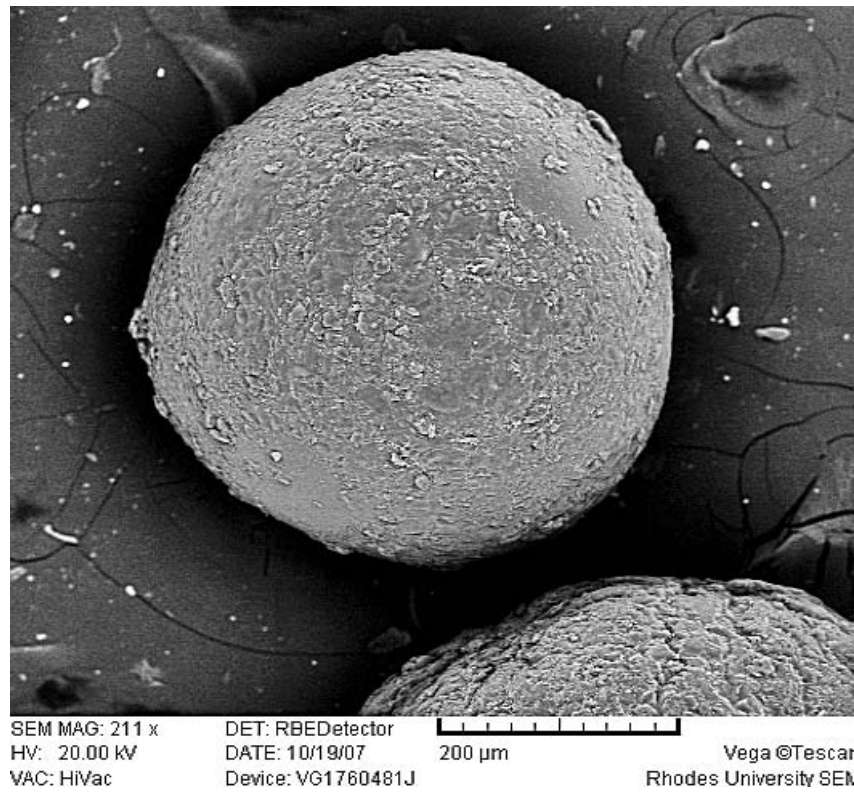

Figure 4. SEM of a PHCl/ Eudragit RL 1:4 (W/W) microcapsule.

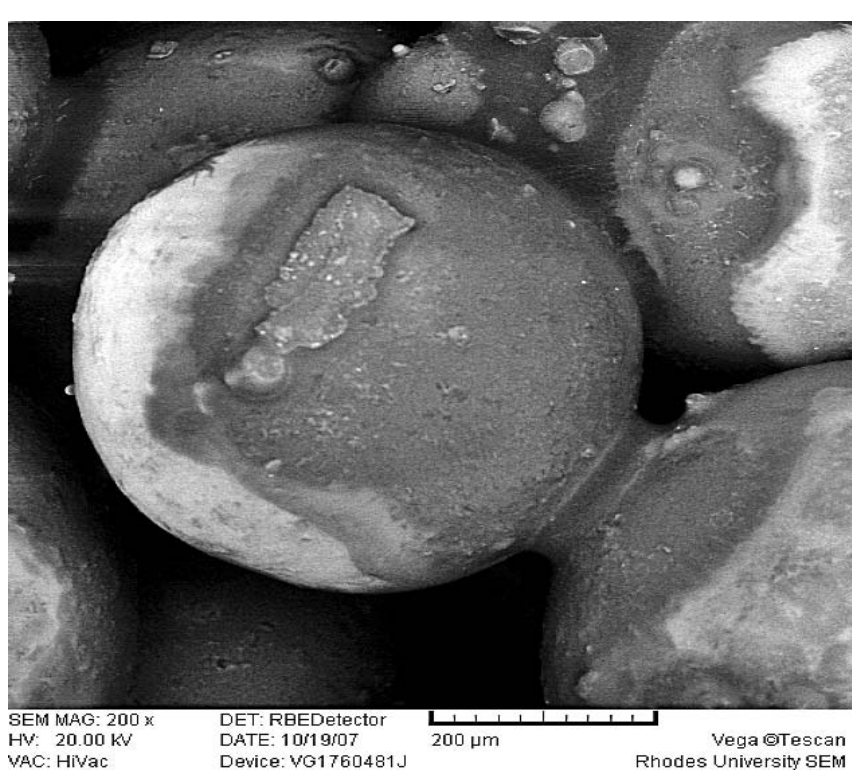

Figure 5. SEM of a VHCI/ Eudragit RL 1:4 (w/w) microcapsule.

surfaces, probably due to the high concentration of drug in the microcapsules.

SEM was performed to determine whether microcapsules had been formed. The use of SEM is important for establishing the encapsulating ability of different polymers, since the degree of porosity may be observed, and therefore, encapsulation ability of the polymers can be 


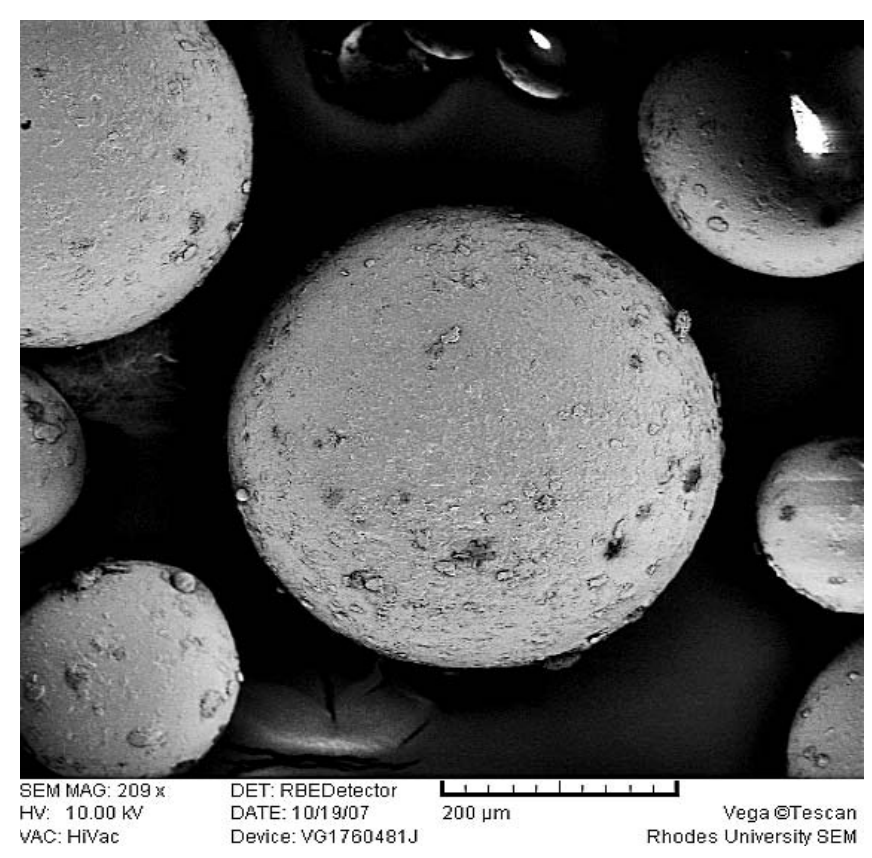

Figure 6. SEM of a PHCl/ Eudragit RS 1:4 (w/w) microcapsule.

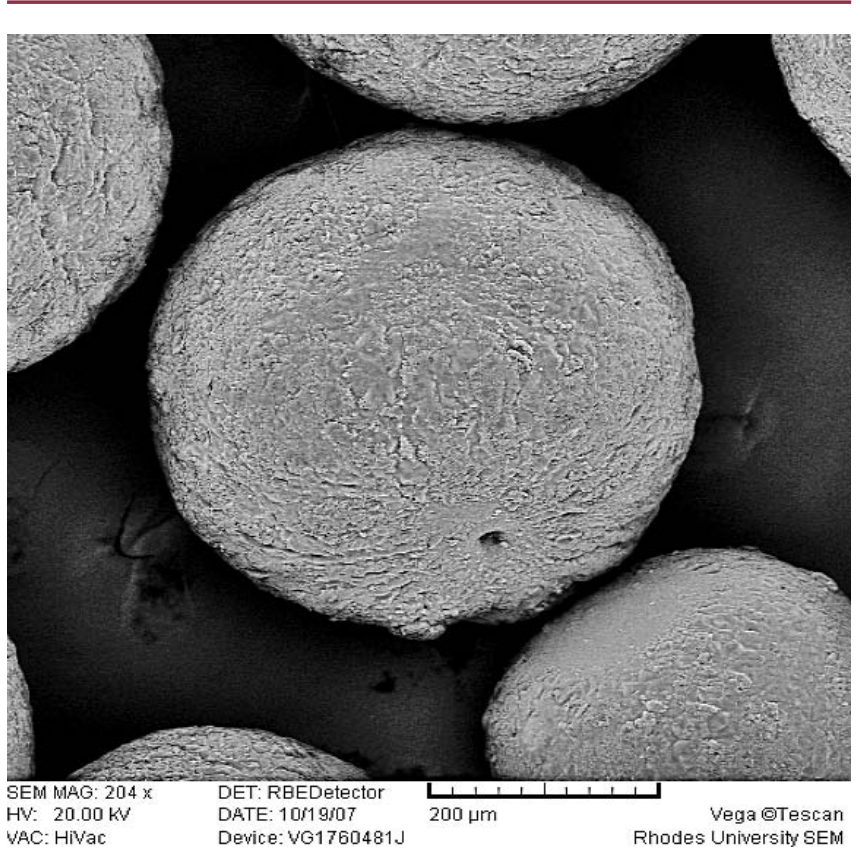

Figure 7. SEM of a VHCl/ Eudragit RS 1:4 (w/w) microcapsule.

established in a qualitative manner. The formulations in which the drug/polymer ratio was 1:4 (w/w) produced uniform spherical particles that were harder to the touch than the ones manufactured at lower drug/polymer concentrations. The presence of residual mineral oil adsorbed onto the surface of the microcapsules, evident in Figure 5 , is a consequence of inadequate washing of the particles with $n$-hexane during manufacture. The surface of the spheres appeared to become smoother with an increase in drug/polymer ratio for $\mathrm{PHCl}$ and $\mathrm{VHCl}$.

Liquid paraffin was selected as a bulk or outer phase, since $\mathrm{PHCl}, \mathrm{VHCl}$, and Eudragit $\mathrm{RS} / \mathrm{RL}$ are only very slightly soluble in liquid paraffin. Acetone has a dielectric constant of 20.7 and was therefore chosen as the dispersed or inner phase, since solvents with dielectric constants between 10 and 40 show poor miscibility with liquid paraffin $(32,33)$. Microspheres that were formulated with low concentrations of Eudragit RL and RS were irregular, non-spherical, soft, and had poor flowability; therefore, they were not considered suitable for analysis.

The dissolution of $\mathrm{PHCl}$ and $\mathrm{VHCl}$ from the microspheres prepared with a 1:4 (w/w) drug/polymer ratio is depicted in Figure 8. Examination of the release profiles reveals that drug release was generally faster for $\mathrm{VHCl}$ and $\mathrm{PHCl}$ microspheres produced with the RL polymer despite the apparent similarity in particle size of the microcapsules. RS and $\mathrm{RL}$ are copolymers of partial esters of acrylic and methacrylic acids containing low amounts of quaternary ammonium groups, approximately $5 \%$ and $10 \%$ for RS and $\mathrm{RL}$, respectively (6). The $\mathrm{RS}$ polymer is water-insoluble, and drug delivery systems prepared from it show $\mathrm{pH}$-independent sustained drug release, attributed to the quaternary ammonium groups (5). The quaternary ammonium groups in the RS and RL chemical structures play an important role in controlling drug release because they relate to water uptake followed by the swelling of the polymers (34). This is most likely because the number of quaternary ammonium groups of $\mathrm{RS}$ is lower than that of $\mathrm{RL}$, which renders $\mathrm{RS}$ less permeable.

There was no significant difference between the dissolution profiles of $\mathrm{PHCl}$ and $\mathrm{VHCl}$ for the same polymer, since the solubility of each of the drugs is similar. The solubility of $\mathrm{PHCl}$ is greater than $150 \mathrm{mg} / \mathrm{mL}$ (35), and the solubility of $\mathrm{VHCl}$ is $123 \mathrm{mg} / \mathrm{mL}$ (19).

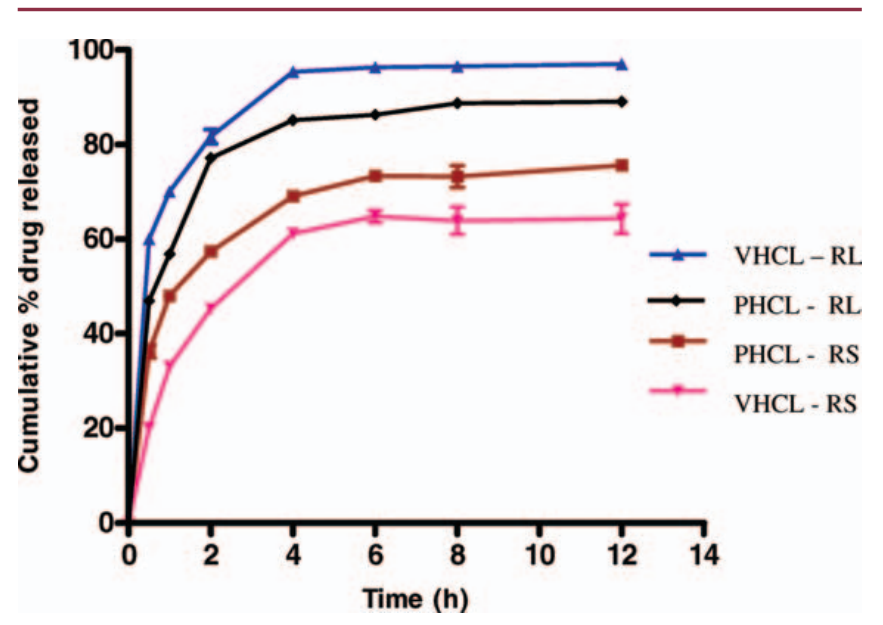

Figure 8. Cumulative percent drug released reported as mean $\pm S D(n=3)$. 
Table 2. Results of Model Fitting of the Release Profiles

\begin{tabular}{|c|c|c|c|c|c|c|c|c|c|c|c|c|c|c|c|c|c|c|c|}
\hline \multirow[b]{2}{*}{ Formulation } & \multicolumn{3}{|c|}{ Zero-order } & \multicolumn{3}{|c|}{ Higuchi } & \multicolumn{4}{|c|}{ Korsmeyer-Peppas } & \multicolumn{4}{|c|}{ Kopcha } & \multicolumn{5}{|c|}{ Makoid-Banakar } \\
\hline & $\mathbf{R}^{2}$ & $k_{o}$ & SSR & $\mathbf{R}^{2}$ & $\boldsymbol{k}_{H}$ & SSR & $\mathbf{R}^{2}$ & $\boldsymbol{k}_{K P}$ & $n$ & SSR & $\mathbf{R}^{2}$ & $A$ & B & SSR & $\mathbf{R}^{2}$ & $\boldsymbol{k}_{M B}$ & $n$ & c & SSR \\
\hline $\mathrm{PHCl}$ & 0.663 & 4.941 & 1951 & 0.861 & 20.02 & 757.2 & 0.920 & 34.97 & 0.314 & 464.6 & 0.869 & 20.04 & 0.0001 & 757.3 & 0.993 & 35.63 & 0.627 & 0.0286 & 40.19 \\
\hline $\mathrm{VHCl}$ & 0.561 & 5.313 & 3473 & 0.790 & 22.31 & 1659 & 0.981 & 59.42 & 0.296 & 63.01 & 0.924 & 49.42 & 0.0001 & 250.7 & 0.981 & 59.10 & 0.293 & 0.0000 & 63.35 \\
\hline
\end{tabular}

The $\mathrm{VHCl}$ and $\mathrm{PHCl} \mathrm{RL}$ microcapsules showed fast release; about $60 \%$ drug was released within the first hour. In the course of our investigation, it was observed that microcapsules were released through the wire mesh basket. It is important to note that the dissolution behavior of granules and powders is greatly influenced by their wettability, surface area, and particle size distribution (26), and thus release of drugs from microcapsules would be affected in a similar manner. In vitro release testing constitutes one of the physicochemical parameters needed to characterize the product.

Drug release from microcapsules should theoretically be slower as the amount of polymer is increased because of an increase in the path length through which the drug has to diffuse.

The total cumulative quantity of drug released at the end of the 12-h dissolution test was below $100 \%$ for all dosage forms. This may in part be due to the relatively slow erosion of the matrix under these test conditions, with a resultant slow release of entrapped drug from the matrices undergoing testing.

The curvilinear nature of the cumulative percentage drug released versus time plots suggest that drug release from the microcapsules did not follow zero-order kinetics. This is confirmed by low correlation coefficients obtained in all cases when these data were fitted to a zero-order model; data are summarized in Table 2. The in vitro dissolution study confirmed the Higuchi-order release pattern. Dissolution data again fit the Kopcha matrix model. However, the mathematical expression that best describes drug release from these microcapsules is the Makoid-Banakar model, with resultant $R^{2}$ values greater than 0.98. The Korsmeyer-Peppas release exponent, $n$, is about 0.3 , which confirms that diffusion is the controlling factor in drug release. This was further established as the ratio of exponents $A / B$ derived from the Kopcha model is greater than 1.The Kopcha model can easily be used to help quantify the contribution of diffusion and polymer relaxation. As seen from the data in Table 2, the value of $A$ is far greater than the value of $B$, which suggests that drug release occurred mainly as a result of Fickian diffusion. The mathematical expression that best describes the release is the Makoid-Banakar model. When the parameter $c$ of the Makoid-Banakar model is equal to zero, this model becomes the Korsmeyer-Peppas power law $\left(\mathrm{e}^{-0 t}=1\right)$.
A relatively high encapsulation efficiency was observed for all microsphere formulations. The encapsulation efficiency was greater than $80 \%$ for all drug candidates investigated, and therefore, it is evident that both the RS and RL polymers are potentially useful materials for the encapsulation of relatively hydrophilic compounds such as $\mathrm{PHCl}$ and $\mathrm{VHCl}$.

The good flow properties $(H R=1.2)$ suggest that the microspheres can be easily handled during processing. One of the challenges was the formation of gas bubbles around the microcapsules that might have affected the results of the dissolution test.

\section{CONCLUSION}

The solvent-evaporation method using Eudragit polymers at optimum levels was effective for the formation of $\mathrm{PHCl}$ and $\mathrm{VHCl}$ microspheres. The $\mathrm{PHCl}$ and $\mathrm{VHCl}$ microcapsules prolonged drug release for about $8 \mathrm{~h}$. The Eudragit polymers are water-insoluble materials, and therefore, $\mathrm{PHCl}$ and $\mathrm{VHCl}$ are not miscible with the polymer and were preferentially encapsulated.

The application of USP Apparatus 1 for the assessment of the Eudragit microcapsules prepared by solvent evaporation was successful, and differences in drug release due to formulation effects were observed. This data set presents evidence that the two BCS Class I drug candidates behave in a similar fashion during formulation development and assessment.

\section{ACKNOWLEDGMENTS}

The authors would like to thank the NRF (RBW), Rhodes University (SMMK, RBW), and Andrew Mellon Foundation (SMMK) for financial support. The Dow Chemical Company, Röhm Pharma, Noveon, Colorcon, and Aspen Pharmacare (Port-Elizabeth, SA) are gratefully acknowledged for their donation of excipients and raw materials.

\section{REFERENCES}

1. Bakan, J. A. Microencapsulation. In The Theory and Practice of Industrial Pharmacy, 3rd ed.; Lachman, L., Lieberman, H. A., Kanig, J. L., Eds.; Lea and Febiger: Philadelphia, PA, 1986; $\mathrm{p} 412$.

2. Hawlader, M. N. A.; Uddin, M. S.; Khin, M. M. Microencapsulated PCM thermal-energy storage system. Appl. Energ. 2003, 74 (1), 195-202. 
3. Wieland-Berghausen, S.; Schote, U.; Frey, M.; Schmidt, F. Comparison of microencapsulation techniques for the water-soluble drugs nitenpyram and clomipramine $\mathrm{HCl}$.J. Controlled Release 2002, 85 (1), 35-43.

4. Yamuda, T.; Onishi, H.; Machida, Y. Sustained release ketoprofen microparticles with ethyl cellulose and carboxymethyl ethyl cellulose. J. Controlled Release 2001, 75 (3), 271-282.

5. Bolourtchian, N.; Karimi, K.; Aboofazeli, R. Preparation and characterization of ibuprofen microspheres. J.Microencap. 2005, 22 (5), 529-538.

6. Haznedar, S.; Dortunç, B. Preparation and in vitro evaluation of Eudragit microspheres containing acetazolamide. Int. J. Pharm. 2004, 269 (1), 131-140.

7. Horoz, B. B.; Kiliic, M.; Arslan, N.; Baykara, Y.T. Effect of different dispersing agents on the characteristics of Eudragit microspheres prepared by a solvent evaporation method. J.Microencap. 2004, 21 (2), 191-202.

8. Dong, W.; Bodmeier, R. Encapsulation of lipophilic drugs within enteric microparticles by a novel coacervation method. Int. J. Pharm. 2006, 326 (1-2), 128-138.

9. Eudragit RS and Eudragit RL data sheets, 1991. Röhm Pharma GmbH, Darmstadt.

10. Dissolution Testing of Immediate Release Solid Oral Dosage Forms; Guidance for Industry; U.S. Department of Health and Human Services, Food and Drug Administration, Center for Drug Evaluation and Research (CDER), U.S. Government Printing Office: Washington, DC, 1997. http://www.fda.gov/cder/ guidance/1713bp1.pdf (accessed Mar 19, 2009).

11. Qureshi, S. A.; McGilveray, I. J. A critical assessment of the USP dissolution apparatus suitability test criteria. Drug Dev. Ind. Pharm. 1995, 21 (8), 905-924.

12. Pandit, J. K.; Singh, S.; Muthu, M. S. Controlled release formulations in neurology practice. Ann. Ind. Acad. Neuro. 2006, 9 (4), 207-216.

13. Amidon, G. L.; Lennernas, H.; Shah, V.P.; Crison, J. R. A theoretical basis for a biopharmaceutic drug classification: the correlation of in vitro drug product dissolution and in vivo bioavailability. Pharm. Res. 1995, 12 (3), 413-420.

14. Eisenberg, M. J.;Brox, A.; Bestawros, A. N. Calcium channel blockers: an update. Am.J.Med. 2004, 116 (1), 35-43.

15. Parfitte, K., Ed. Martindale: The Complete Drug Reference, 32nd ed.; The Pharmaceutical Press: London, 1999; p 937.

16. Lindenberg, M.; Kopp, S.; Dressman, J. B. Classification of orally administered drugs on the World Health Organization Model list of Essential Medicines according to the biopharmaceutics classification system. Eur. J. Pharm. Biopharm. 2004, 58 (2), 265-278.

17. Mukherjee, B.; Mahnti, B.; Panda, P.; Mahapatra, S. Preparation and evaluation of verapamil hydrochloride microcapsules. Am. J. Ther. 2005, 12 (5), 417-424.
18. Goracinova, K.; Glavas, M.; Kumbaradi, E. F.; Mladenovska, K. Preparation and characterisation of microcapsules with verapamil hydrochloride. Acta Pharmaceut. 2000, 50 (1), 57-63.

19. Sipahigil, O.; Dortunç, B. Preparation and in vitro evaluation of verapamil $\mathrm{HCl}$ and ibuprofen containing carrageenan beads. Int. J. Pharm. 2001, 228 (1-2), 119-128.

20. Manekar, N. C.; Puranik, P. K.; Joshi, S. B. Microencapsulation of propranolol hydrochloride by the solvent evaporation technique. J.Microencap. 1992, $9(1), 63-66$.

21. Chiao, C. S. L.; Price, J. C. Formulation, preparation and dissolution characteristics of propranolol hydrochloride microspheres. J. Microencap. 1994, 11 (2), 153-159.

22. Jones, D. S.; Pearce, K. J. An investigation of the effects of some process variables on the microencapsulation of propanolol hydrochloride by the solvent evaporation method. Int. J. Pharm. 1995, 118 (2), 199-205.

23. Kumar, V.; Medina, M. L. R.; Yang, D. Preparation, Characterization, and Tableting Properties of a New Cellulose-based Pharmaceutical Aid.Int. J. Pharm. 2002, 235 (1-2), 129-140.

24. Karasulu, H.Y.;Taneri, F.; Sanal, E.; Güneri, T.; Ertan, G. Sustained release bioadhesive effervescent ketoconazole microcapsules tabletted for vaginal delivery. J. Microencap. 2002, 9 (3), 357-362.

25. Donbrow, M.; Samuelov, Y. Zero order drug delivery from double-layered porous films: release rate profiles from ethyl cellulose, hydroxypropylcellulose and polyethylene glycol mixtures.J. Pharm. Pharmacol. 1980, 32 (7), 463-470.

26. Higuchi, T. Rate of release of medicaments from ointment bases containing drugs in suspension. J. Pharm. Sci. 1961, 50 (10), 874-875.

27. Higuchi, T. Mechanism of sustained-action medication. Theoretical analysis of rate of release of solid drugs dispersed in solid matrices. J. Pharm. Sci. 1963, 52 (12), 1145-1149.

28. Korsmeyer, R.W.; Gurny, R.; Doelker, E. M.; Buri, P.; Peppas, N. A. Mechanism of solute release from porous hydrophilic polymers. Int. J. Pharm. 1983, 15 (1), 25-35.

29. Kopcha, M.; Lordi, N.; Tojo, K. J. Evaluation of release from selected thermosoftening vehicles. J. Pharm. Pharmacol. 1991, 43 (6), 382-387.

30. Pais, J.Intuiting mathematical objects using kinetigrams. J. Online Math. Appl. (JOMA) 2001, 1 (2). http://www.joma.org/jsp/search/searchResults. jsp?url=http://mathdl.maa.org/mathDL/4/ ?pa $=$ content $\&$ sa $=$ viewDocument $\&$ nodeld $=429$ (accessed Mar 19, 2009).

31. El-Kamel, A.; Al-Shora, D. H.; El-Sayed, Y. M. Formulation and pharmacodynamic evaluation of captopril sustained release microcapsules. J. Microencap. 2006, 23 (4), 389-404.

32. Sengel, C. T.; Hascicek, C.; Gonul, N. Development and in-vitro evaluation of modified release tablets 
including ethylcellulose microspheres loaded with diltiazem hydrochloride. J. Microencap. 2006, 23 (2), 135-152.

33. Mateovic, T.; Kriznar, B.; Bogataj, M.; Mrhar, A. The influence of stirring rate on biopharmaceutical properties of Eudragit RS microspheres. J. Microencap. 2002, 19 (1), 29-36.

34. Fujimori, J.;Yoshihashi, Y.; Yonemochi, E.; Terada, K. Application of Eudragit RS to thermo-sensitive drug delivery systems II. Effect of temperature on drug permeability through membrane consisting of Eudragit RS/PEG 400 blend polymers. J. Controlled Release 2005, 102 (1), 49-57.

35. Ubrich, N.; Bouillot, P.; Pellerin, C.; Hoffman, M.; Maincent, P. Preparation and characterization of propranolol hydrochloride nanoparticles: a comparative study. J. Controlled Release 2004, 97 (2), 291-300. 